PROCEEDINGS OF THE

AMERICAN MATHEMATICAL SOCIETY

Volume 128, Number 10, Pages 2933-2941

S 0002-9939(00)05634-3

Article electronically published on April 7, 2000

\title{
COMPACTNESS OF FLOQUET ISOSPECTRAL SETS FOR THE MATRIX HILL'S EQUATION
}

\author{
ROBERT CARLSON
}

(Communicated by Hal L. Smith)

\begin{abstract}
Let $\mathcal{M}(Q)$ denote the set of self adjoint $K \times K$ potentials for the matrix Hill's equation having the same Floquet multipliers as $-D^{2}+Q$. Elementary methods are used to show that $\mathcal{M}(Q)$ has compact closure in the space of continuous matrix valued functions.
\end{abstract}

\section{INTRODUCTION}

This paper considers some questions of inverse spectral theory for the matrix Hill's equation

$$
-Y^{\prime \prime}+Q(x) Y=\lambda Y, \quad Y \in \mathbf{C}^{K}, \quad \lambda \in \mathbf{C}, \quad Q(x)=Q(x+1) .
$$

The $K \times K$ matrix function $Q: \mathbf{R} \rightarrow M_{K}(\mathbf{C})$ is assumed to have $J \geq 0$ continuous derivatives. The basic Floquet theory may be described as in the scalar case. For each $\lambda \in \mathbf{C}$ the equation (1) has a $2 K$ dimensional space of solutions on which translation by 1 acts linearly. The Floquet multipliers $\xi_{k}(\lambda)$ are the eigenvalues of this linear map.

Introduce the $K \times K$ matrix solutions $C(x, \lambda), S(x, \lambda)$ of (1) with the initial data

$$
C(0, \lambda)=I_{K}, \quad C^{\prime}(0, \lambda)=0_{K}, \quad S(0, \lambda)=0_{K}, \quad S^{\prime}(0, \lambda)=I_{K} .
$$

With respect to the basis given by the columns of $C(x, \lambda)$ and $S(x, \lambda)$ the $2 K \times 2 K$ matrix

$$
\Psi_{1}=\left(\begin{array}{cc}
C(1, \lambda) & S(1, \lambda) \\
C^{\prime}(1, \lambda) & S^{\prime}(1, \lambda)
\end{array}\right)
$$

represents the translation operator. The Floquet variety $\mathcal{F}_{Q}$ consists of the pairs $(\lambda, \xi) \in \mathbf{C}^{2}$ satisfying the characteristic polynomial $\operatorname{det}\left(\xi I-\Psi_{1}(\lambda)\right)=0$.

Hill's equation (1) will have periodic, respectively antiperiodic, eigenvalues $\lambda_{n}$ where at least one of the multipliers $\xi_{k}\left(\lambda_{n}\right)$ is 1 , respectively -1 . In the scalar case $(K=1)$ the set $\mathcal{M}(Q)$ is usually defined to be the set of potentials having the same periodic and antiperiodic spectra as $Q$. By the Wronskian identity the determinant of the translation map is 1 , so the eigenvalues in the scalar case may be described using the discriminant $\Delta(\lambda)=C(1, \lambda)+S^{\prime}(1, \lambda)$, which is just the trace of the translation map. The periodic and antiperiodic eigenvalues are respectively the roots of $\Delta-2$ and $\Delta+2$.

Received by the editors November 10, 1998.

2000 Mathematics Subject Classification. Primary 34A55; Secondary 34L40.

Key words and phrases. Hill's equation, inverse spectral theory, KdV. 
Since the discriminant is an entire function with order of growth $1 / 2$ [9, p. 20], Hadamard's Theorem [1, p. 206] implies that $\Delta(\lambda)$ may be recovered up to a constant factor from the periodic or antiperiodic eigenvalues. The constant may also be determined [9, p. 22]. Thus in the scalar case being isospectral for periodic eigenvalues is equivalent to having the same set of Floquet multipliers for all $\lambda \in \mathbf{C}$. This Floquet isospectrality is the condition we will consider in the matrix case.

The development of the elaborate and beautiful inverse spectral theory for the scalar Hill's equation [5, 10, 11, 13] received a strong impetus from the discovery [8] that solutions of the $\mathrm{KdV}$ equation with initial data $Q$ evolve in $\mathcal{M}(Q)$. As in the scalar case, the matrix equation

$$
Q_{t}=-(1 / 4) Q^{\prime \prime \prime}+(3 / 4) Q Q^{\prime}+(3 / 4) Q^{\prime} Q
$$

may be expressed in the Lax form $\frac{d L}{d t}=[L, A]$, and spatially periodic solutions of the nonlinear evolution equation lead to Floquet isospectral operators $L(t)$.

Except for [2, which considers spectral theoretic characterizations of constant matrix potentials, it appears that almost none of the inverse spectral theory for the matrix Hill's equation has been developed. The main goal of this work is to establish the compactness result for $\mathcal{M}(Q)$ contained in Theorem[1.1. To be precise about the differentiability of the potentials, define

$$
\mathcal{Q}=\left\{Q: \mathbf{R} \rightarrow M_{K}(\mathbf{C}) \mid Q(x) \in C^{5}(\mathbf{R}), Q(x)=Q(x+1)\right\} .
$$

Theorem 1.1. The set of self adjoint potentials $Q(x) \in \mathcal{Q}$ with the same Floquet variety has compact closure in the space of continuous $K \times K$ matrix valued functions.

The limited results currently available for matrix potentials contrast sharply with the detailed analyses of the scalar case. More refined compactness results were used already in 8. Generalizing earlier results for finite gap potentials, McKean and Trubowitz [1] proved that the set of $C^{\infty}$ scalar potentials with a fixed periodic spectrum is a product of (generally infinitely many) circles. By an explicit construction, this result is extended to potentials in $L^{2}[0,1]$ in $[3$. Alternate methods [6] provided a similar result for potentials with a square integrable derivative. In [7] the $L^{2}$ potentials are given a global set of coordinates which simultaneously exhibit all isospectral sets as products of circles. A thorough analysis of isospectral sets for potentials in $L^{2}[0,1]$ with a variety of generalized periodic boundary conditions is in [12].

\section{Preliminary Results}

Estimates describing solutions of the equation (11) as $|\lambda| \rightarrow \infty$ may be established using techniques commonly seen in the scalar case. Except for some minor notational changes, and the rearrangement of some terms which do not commute in the vector case, our estimates and their proofs are very similar to those appearing in [4]. The reader may consult this reference for the proofs of Lemma 2.1] and Lemma 2.2

We begin by establishing some notational conventions. For $\lambda \in \mathbf{C}$ let $\omega=\sqrt{\lambda}$, the root chosen continuously for $-\pi<\arg (\lambda) \leq \pi$ and positive for $\lambda>0$. The 
imaginary part of $\omega$ is denoted by $\Im \omega$. A element $Y \in \mathbf{C}^{K}$ will have the usual norm

$$
|Y|=\left[\sum_{k=1}^{K}\left|y_{k}\right|^{2}\right]^{1 / 2}, \quad Y=\left(\begin{array}{c}
y_{1} \\
\vdots \\
y_{K}
\end{array}\right)
$$

and a $K \times K$ matrix $Q$ will have the operator norm

$$
\|Q\|=\sup _{|Y|=1}|Q Y| .
$$

The $K \times K$ identity matrix is $I_{K}$, and the zero matrix is $0_{K}$.

Estimates for solutions of (11) may be developed by using the model equation $-Y^{\prime \prime}=\lambda Y$. This equation has a basis of $2 K$ solutions which are the columns of the $K \times K$ diagonal matrix valued functions $\cos (\omega x) I_{K}, \omega^{-1} \sin (\omega x) I_{K}$. By using the variation of parameters formula a solution of (1) satisfying $Y(0, \lambda)=\alpha$, $Y^{\prime}(0, \lambda)=\beta$, with $\alpha, \beta \in \mathbf{C}^{K}$, may be expressed as a solution of the integral equation

$$
Y(x, \lambda)=\cos (\omega x) \alpha+\omega^{-1} \sin (\omega x) \beta+\omega^{-1} \int_{0}^{x} \sin (\omega[x-t]) Q(t) Y(t, \lambda) d t .
$$

Differentiation with respect to $x$ gives

$$
Y^{\prime}(x, \lambda)=-\omega \sin (\omega x) \alpha+\cos (\omega x) \beta+\int_{0}^{x} \cos (\omega[x-t]) Q(t) Y(t, \lambda) d t .
$$

An iteration scheme based on (21) and integration by parts provides the desired expansion of $Y(x, \lambda)$, together with error estimates, when $Q(x)$ is sufficiently differentiable. For $J \geq 0$ let $C^{J}$ denote the Banach space of $K \times K$ matrix valued functions $Q(x)$ whose components have $J$ continuous derivatives on $[0,1]$. Equip $C^{J}$ with the norm

$$
\|Q\|_{J}=\max \left\|Q^{(j)}(x)\right\|, \quad 0 \leq j \leq J, \quad 0 \leq x \leq 1 .
$$

It will be notationally convenient to define $\mathcal{A}_{j}(x)=Q(x) A_{j}(x)$ and $\mathcal{B}_{j}(x)=$ $Q(x) B_{j}(x)$.

Lemma 2.1. Suppose that $Q \in C^{J}$, and $Y(x, \lambda)$ is the solution of (1) satisfying $Y(0, \lambda)=\alpha$ and $Y^{\prime}(0, \lambda)=\beta$, with $|\alpha|+|\beta| \leq 1,0 \leq x \leq 1$, and $|\omega| \geq 1$. Then there are $\mathbf{C}^{K}$ valued functions $A_{j}(x)$ and $B_{j}(x)$, such that

$$
\left|Y(x, \lambda)-\sum_{j=0}^{J} \omega^{-j}\left[\cos (\omega x) A_{j}(x)+\sin (\omega x) B_{j}(x)\right]\right|=O\left(\omega^{-J-1} e^{|\Im \omega| x}\right) .
$$

For $0 \leq j \leq J$ the coefficients $A_{j}(x), B_{j}(x)$ satisfy

$$
\begin{gathered}
A_{0}(x)=\alpha, \quad A_{1}(x)=0, \\
A_{2}(x)=2^{-2}[Q(x)-Q(0)] \alpha-2^{-1} \int_{0}^{x} Q(t)\left[\beta+2^{-1} \int_{0}^{t} Q(s) \alpha d s\right] d t, \\
B_{0}(x)=0, \quad B_{1}(x)=\beta+2^{-1} \int_{0}^{x} Q(t) \alpha d t, \quad B_{2}(x)=0,
\end{gathered}
$$


and for $j \geq 3$ the coefficients satisfy the recursion relations

$$
\begin{gathered}
A_{j}(x)=\sum_{k=0}^{\lfloor(j-2) / 2\rfloor}(-1)^{k} 2^{-2 k-2}\left[\mathcal{A}_{j-2 k-2}^{(2 k)}(x)-\mathcal{A}_{j-2 k-2}^{(2 k)}(0)\right] \\
-2^{-1} \int_{0}^{x} \mathcal{B}_{j-1}(t) d t+\sum_{k=0}^{\lfloor(j-3) / 2\rfloor}(-1)^{k} 2^{-2 k-3}\left[\mathcal{B}_{j-2 k-3}^{(2 k+1)}(x)-\mathcal{B}_{j-2 k-3}^{(2 k+1)}(0)\right], \\
B_{j}(x)=-\sum_{k=0}^{\lfloor(j-3) / 2\rfloor}(-1)^{k} 2^{-2 k-3}\left[\mathcal{A}_{j-2 k-3}^{(2 k+1)}(x)+\mathcal{A}_{j-2 k-3}^{(2 k+1)}(0)\right] \\
+2^{-1} \int_{0}^{x} \mathcal{A}_{j-1}(t) d t+\sum_{k=0}^{\lfloor(j-2) / 2\rfloor}(-1)^{k} 2^{-2 k-2}\left[\mathcal{B}_{j-2 k-2}^{(2 k)}(x)+\mathcal{B}_{j-2 k-2}^{(2 k)}(0)\right] .
\end{gathered}
$$

The function $Y^{\prime}(x, \lambda)$ has a similar expansion obtained from that of $Y(x, \lambda)$ by termwise differention.

Lemma 2.2. Under the hypotheses of Lemma 2.1 there are $\mathbf{C}^{K}$ valued functions $C_{j}(x)$ and $D_{j}(x)$ such that

$$
\left|Y^{\prime}(x, \lambda)-\sum_{j=-1}^{J-1} \omega^{-j}\left[\cos (\omega x) C_{j}(x)+\sin (\omega x) D_{j}(x)\right]\right|=O\left(\omega^{-J} e^{|\Im \omega| x}\right) .
$$

The coefficients $C_{j}(x), D_{j}(x)$ satisfy $C_{-1}(x)=0, D_{-1}(x)=-\alpha$, and

$$
C_{j}(x)=A_{j}^{\prime}(x)+B_{j+1}(x), \quad D_{j}(x)=B_{j}^{\prime}(x)-A_{j+1}(x), \quad j=0, \ldots, J-1 .
$$

From the computed initial coefficients and the recursion relations we can see that half of the coefficients vanish.

Lemma 2.3. If $j$ is odd, the coefficients $A_{j}(x)$ and $C_{j}(x)$ are 0 . If $j$ is even, the coefficients $B_{j}(x)$ and $D_{j}(x)$ are 0 .

The asymptotic expansions for $Y(x, \lambda)$, and $Y^{\prime}(x, \lambda)$ specialize to give expansions for the matrix functions $C(1, \lambda), C^{\prime}(1, \lambda), S(1, \lambda)$, and $S^{\prime}(1, \lambda)$. Defining $Q_{0}=$ $\int_{0}^{1} Q(t) d t$, and using the periodic continuity condition $Q(1)=Q(0)$, the following formulas are obtained for $J \geq 2$ :

$$
\begin{gathered}
C(1, \lambda)=\cos (\omega) I_{K}+2^{-1} \omega^{-1} \sin (\omega) Q_{0} \\
-2^{-2} \omega^{-2} \cos (\omega) \int_{0}^{1} Q(t) \int_{0}^{t} Q(s) d s d t+O\left(\omega^{-3} e^{|\Im \omega|}\right) \\
S(1, \lambda)=\omega^{-1} \sin (\omega) I_{K}-2^{-1} \omega^{-2} \cos (\omega) Q_{0} \\
+2^{-2} \omega^{-3} \sin (\omega)\left[2 Q(0)-\int_{0}^{1} Q(t) \int_{0}^{t} Q(s) d s d t\right]+O\left(\omega^{-4} e^{|\Im \omega|}\right), \\
C^{\prime}(1, \lambda)=-\omega \sin (\omega) I_{K}+2^{-1} \cos (\omega) Q_{0} \\
+2^{-2} \omega^{-1} \sin (\omega)\left[2 Q(0)+\int_{0}^{1} Q(t) \int_{0}^{t} Q(s) d s d t\right]+O\left(\omega^{-2} e^{|\Im \omega|}\right),
\end{gathered}
$$




$$
\begin{gathered}
S^{\prime}(1, \lambda)=\cos (\omega) I_{K}+2^{-1} \omega^{-1} \sin (\omega) Q_{0} \\
-2^{-2} \omega^{-2} \cos (\omega) \int_{0}^{1} Q(t) \int_{0}^{t} Q(s) d s d t+O\left(\omega^{-3} e^{|\Im \omega|}\right) .
\end{gathered}
$$

\section{Trace of the Floquet matrix}

The above expansions may be used to develop asymptotic expressions for $\operatorname{tr} \Psi_{1}(\lambda)$. Since the trace is independent of the selected basis, the coefficients of these expansions depend only on the set of Floquet eigenvalues. An analysis of these coefficients provides the proof of Theorem 1.1] It will be convenient to assume that the matrix $Q_{0}$ is diagonal, $Q_{0}=\operatorname{diag}\left[q_{1}, \ldots, q_{K}\right]$. Of course a simple linear change of variables reduces (11) to this case when $Q_{0}$ is similar to a diagonal matrix.

Rather than working directly with $\Psi_{1}(\lambda)$ it is easier to consider the similar matrix

$$
\Psi(\lambda)=\left(\begin{array}{cc}
C(1, \lambda) & \omega S(1, \lambda) \\
\omega^{-1} C^{\prime}(1, \lambda) & S^{\prime}(1, \lambda)
\end{array}\right)=\left(\begin{array}{cc}
I_{K} & 0_{K} \\
0_{K} & \omega^{-1} I_{K}
\end{array}\right) \Psi_{1}(\lambda)\left(\begin{array}{cc}
I_{K} & 0_{K} \\
0_{K} & \omega I_{K}
\end{array}\right)
$$

whose entries have a more uniform description in the style of (3). We may write

$$
\Psi(\lambda)=\sum_{j=0}^{J} \psi_{j}(\lambda)+O\left(\omega^{-J-1} e^{|\Im \omega|}\right),
$$

with

$$
\psi_{j}(\lambda)=2^{-j} \omega^{-j}\left[\psi_{j}^{A} \cos (\omega)+\psi_{j}^{B} \sin (\omega)\right],
$$

where the coefficients $\psi_{j}^{A}, \psi_{j}^{B}$ are constant $K \times K$ matrices. By Lemma 2.2 the coefficients $C_{j}$ and $D_{j}$ for the solutions $C(x, \lambda)$ and $S(x, \lambda)$ may be computed directly from the coefficients $A_{j}=A_{j}(1, \lambda)$ and $B_{j}=B_{j}(1, \lambda)$, and the derivatives $A_{j}^{\prime}=A_{j}^{\prime}(1, \lambda)$ and $B_{j}^{\prime}=B_{j}^{\prime}(1, \lambda)$. Using the subscripts $C, S$ to indicate the source function $C(x, \lambda)$ or $S(x, \lambda)$, we find that

$$
\begin{aligned}
& 2^{-j} \psi_{j}^{A}=\left(\begin{array}{cc}
A_{C, j} & A_{S, j+1} \\
A_{C, j-1}^{\prime}+B_{C, j} & A_{S, j}^{\prime}+B_{S, j+1}
\end{array}\right), \\
& 2^{-j} \psi_{j}^{B}=\left(\begin{array}{cc}
B_{C, j} & B_{S, j+1} \\
B_{C, j-1}^{\prime}-A_{C, j} & B_{S, j}^{\prime}-A_{S, j+1}
\end{array}\right) .
\end{aligned}
$$

It will be convenient to introduce the following notation:

$$
I_{m}(Q)=\int_{0}^{1} Q\left(t_{1}\right) \int_{0}^{t_{1}} Q\left(t_{2}\right) \cdots \int_{0}^{t_{m-1}} Q\left(t_{m}\right) d t_{m} \ldots d t_{1} .
$$

For the following calculations it is assumed that $Q(x)$ has 5 continuous derivatives on $\mathbf{R}$, so that $Q^{(j)}(0)=Q^{(j)}(1)$ for $j=0, \ldots, 5$. The first few terms in the expansion of $\Psi(\lambda)$ are

$$
\begin{gathered}
\psi_{0}^{A}=\left(\begin{array}{cc}
I_{K} & 0 \\
0 & I_{K}
\end{array}\right), \quad \psi_{0}^{B}=\left(\begin{array}{cc}
0 & I_{K} \\
-I_{K} & 0
\end{array}\right), \\
\psi_{1}^{A}=\left(\begin{array}{cc}
0 & -Q_{0} \\
Q_{0} & 0
\end{array}\right), \quad \psi_{1}^{B}=\left(\begin{array}{cc}
Q_{0} & 0 \\
0 & Q_{0}
\end{array}\right), \\
\psi_{2}^{A}=\left(\begin{array}{cc}
-I_{2}(Q) & 0 \\
0 & -I_{2}(Q)
\end{array}\right), \quad \psi_{2}^{B}=\left(\begin{array}{cc}
0 & 2 Q(0)-I_{2}(Q) \\
2 Q(0)+I_{2}(Q) & 0
\end{array}\right),
\end{gathered}
$$




$$
\begin{aligned}
& \psi_{3}^{A}=\left(\begin{array}{cc}
0 & -Q(0) Q_{0}-Q_{0} Q(0) \\
& -\int_{0}^{1} Q^{2}(t)+I_{3}(Q) \\
-Q(0) Q_{0}-Q_{0} Q(0) & 0 \\
+\int_{0}^{1} Q^{2}(t)-I_{3}(Q) &
\end{array}\right), \\
& \psi_{3}^{B}=\left(\begin{array}{cc}
-2 Q^{\prime}(0)+Q(0) Q_{0}-Q_{0} Q(0) & 0 \\
+\int_{0}^{1} Q^{2}(t)-I_{3}(Q) & \\
0 & 2 Q^{\prime}(0)-Q(0) Q_{0}+Q_{0} Q(0) \\
& +\int_{0}^{1} Q^{2}(t)-I_{3}(Q)
\end{array}\right) \\
& \psi_{4}^{A}= \\
& \left(\begin{array}{cc}
Q_{0} Q^{\prime}(0)+Q^{\prime}(0) Q_{0} & \\
+I_{2}(Q) Q(0)-Q(0) I_{2}(Q) & \\
-\int_{0}^{1} Q(t) \int_{0}^{t} Q^{2}(s)-\int_{0}^{1} Q^{2}(t) \int_{0}^{t} Q(s) & \\
+I_{4}(Q)+\int_{0}^{1} Q(t) Q^{\prime}(t) & -Q^{\prime}(0) Q_{0}-Q_{0} Q^{\prime}(0) \\
0 & +Q(0) I_{2}(Q)-I_{2}(Q) Q(0) \\
& -\int_{0}^{1} Q^{2}(t) \int_{0}^{t} Q(s)-\int_{0}^{1} Q(t) \int_{0}^{t} Q^{2}(s) \\
& +I_{4}(Q)+\int_{0}^{1} Q(t) Q^{\prime}(t)
\end{array}\right) \\
& \begin{array}{c}
\psi_{4}^{B}= \\
\\
\left(\begin{array}{cc}
6 Q^{2}(0)-Q_{0} Q^{\prime}(0)+Q^{\prime}(0) Q_{0} \\
0 & -2 Q^{(2)}(0)-I_{2}(Q) Q(0)-Q(0) I_{2}(Q) \\
& +\int_{0}^{1} Q(t) Q^{\prime}(t)+I_{4}(Q) \\
& -\int_{0}^{1} Q(t) \int_{0}^{t} Q^{2}(s)-\int_{0}^{1} Q^{2}(t) \int_{0}^{t} Q(s) \\
2 Q^{2}(0)+Q^{\prime}(0) Q_{0}-Q_{0} Q^{\prime}(0) & 0 \\
-Q(0) I_{2}(Q)-I_{2}(Q) Q(0) & \\
-2 Q^{(2)}(0)-I_{4}(Q)-\int_{0}^{1} Q(t) Q^{\prime}(t) & \\
+\int_{0}^{1} Q^{2}(t) \int_{0}^{t} Q(s)+\int_{0}^{1} Q(t) \int_{0}^{t} Q^{2}(s) &
\end{array}\right) .
\end{array}
\end{aligned}
$$

Lemma 3.1. Suppose that $Q(x)$ has 5 continuous derivatives on $\mathbf{R}$, and is periodic with period 1 . The following expressions are determined by the Floquet variety $\mathcal{F}_{Q}$ :

$$
\begin{gathered}
\mathcal{I}_{1}=\operatorname{tr}\left[Q_{0}\right], \quad \mathcal{I}_{2}=\operatorname{tr}\left[I_{2}(Q)\right], \quad \mathcal{I}_{3}=\operatorname{tr}\left[\int_{0}^{1} Q^{2}(t) d t-I_{3}(Q)\right], \\
\mathcal{I}_{4}=\operatorname{tr}\left[-\int_{0}^{1} Q(t) \int_{0}^{t} Q^{2}(s)-\int_{0}^{1} Q^{2}(t) \int_{0}^{t} Q(s)+I_{4}(Q)+\int_{0}^{1} Q(t) Q^{\prime}(t)\right],
\end{gathered}
$$

and

$$
\begin{gathered}
\mathcal{I}_{5}=\operatorname{tr}\left[2 \int_{0}^{1}\left[Q^{\prime}(x)\right]^{2}+4 \int_{0}^{1} Q^{3}+Q_{0} \int_{0}^{1} Q(t) Q^{\prime}(t) d t\right. \\
-2 \int_{0}^{1} Q^{2}(x) \int_{0}^{x} Q(t) \int_{0}^{t} Q(s)-2 \int_{0}^{1} Q(x) \int_{0}^{x} Q^{2}(t) \int_{0}^{t} Q(s) \\
\left.-2 \int_{0}^{1} Q(x) \int_{0}^{x} Q(t) \int_{0}^{t} Q^{2}(s)+2 I_{5}(Q)\right] .
\end{gathered}
$$


Proof. The function $\operatorname{tr} \Psi(\lambda)$ depends only on the Floquet multipliers. Taking the trace of (3.a) gives

$$
\operatorname{tr} \Psi(\lambda)=\sum_{j=0}^{J}\left[2^{-j} \omega^{-j} \cos (\omega) \operatorname{tr}\left(\psi_{j}^{A}\right)+2^{-j} \omega^{-j} \sin (\omega) \operatorname{tr}\left(\psi_{j}^{B}\right)\right]+O\left(\omega^{-J-1} e^{|\Im \omega|}\right) .
$$

By virtue of the elementary integral formulas

$$
\begin{gathered}
\int_{1}^{T} \cos ^{2}(\omega) d \omega=\frac{T-1}{2}+\frac{\sin (2 T)-\sin (2)}{4} \\
\int_{1}^{T} \sin (\omega) \cos (\omega) d \omega=\frac{\sin ^{2}(T)-\sin ^{2}(1)}{2}
\end{gathered}
$$

the coefficients $\operatorname{tr}\left(\psi_{j}^{A}\right)$ and $\operatorname{tr}\left(\psi_{j}^{B}\right)$ may be recovered from $\operatorname{tr} \Psi(\lambda)$ by recursively computing

$$
\psi_{j}^{A}=\lim _{T \rightarrow \infty} \frac{2}{T-1} \int_{1}^{T} 2^{j} \omega^{j}\left[\operatorname{tr} \Psi(\lambda)-\sum_{l=0}^{j-1} \operatorname{tr} \psi_{l}(\lambda)\right] \cos (\omega) d \omega,
$$

and

$$
\psi_{j}^{B}=\lim _{T \rightarrow \infty} \frac{2}{T-1} \int_{1}^{T} 2^{j} \omega^{j}\left[\operatorname{tr} \Psi(\lambda)-\sum_{l=0}^{j-1} \operatorname{tr} \psi_{l}(\lambda)\right] \sin (\omega) d \omega .
$$

Consequently, the traces of $\psi_{j}^{A}$ and $\psi_{j}^{B}$ are Floquet spectral invariants. The last in the list of invariants requires the computation of $\operatorname{tr} \psi_{5}^{B}$, an ugly exercise in elementary algebra too long to record here.

Compactness of $\mathcal{M}(Q)$ is established by mixing a standard method [10] with some additional matrix analysis.

Proof of Theorem 1.1. We begin with some elementary matrix identities. A simple computation shows that for $K \times K$ matrices $A$ and $B, \operatorname{tr}[A B]=\operatorname{tr}[B A]$. Applying this identity to a product of $n$ matrices gives

$$
\operatorname{tr}\left[A_{1} \cdots A_{n-1} A_{n}\right]=\operatorname{tr}\left[A_{n} A_{1} \cdots A_{n-1}\right]
$$

which shows that the trace of a product is invariant under cyclic permutation of the factors. Examining the diagonal entries of a product, we also find that

$$
\operatorname{diag}[A B]=\operatorname{diag}\left[(A B)^{T}\right]=\operatorname{diag}\left[B^{T} A^{T}\right]=\overline{\operatorname{diag}\left[B^{*} A^{*}\right]} .
$$

If $A$ and $B$ are self adjoint, then

$$
\operatorname{diag}[A B]=\overline{\operatorname{diag}[B A]}
$$

Analysis of the spectral invariants of Lemma 3.1 begins with

$$
\operatorname{tr}\left[I_{2}(Q)\right]=\operatorname{tr} \int_{0}^{1} Q(t) \int_{0}^{t} Q(s) d s d t=\operatorname{tr} \int_{0}^{1}\left[\int_{0}^{t} Q(s) d s\right] Q(t) d t,
$$

by 3.b. Thus

$$
2 \operatorname{tr}\left[I_{2}(Q)\right]=\operatorname{tr} \int_{0}^{1} \frac{d}{d t}\left[\int_{0}^{t} Q(s) d s\right]^{2} d t=\operatorname{tr}\left[\int_{0}^{1} Q(s) d s\right]^{2}=\operatorname{tr}\left[Q_{0}^{2}\right] .
$$


The invariant $\mathcal{I}_{3}$ contains $I_{3}(Q)$. Integration by parts gives

$$
I_{3}(Q)=Q_{0} I_{2}(Q)-\int_{0}^{1}\left[\int_{0}^{t} Q(s)\right]\left[Q(t) \int_{0}^{t} Q(s) d s\right] d t .
$$

Using (3.b) gives

$$
3 \operatorname{tr} \int_{0}^{1}\left[\int_{0}^{t} Q(s)\right]\left[Q(t) \int_{0}^{t} Q(s) d s\right] d t=\operatorname{tr} \int_{0}^{1} \frac{d}{d t}\left[\int_{0}^{t} Q(s)\right]^{3} d t=\operatorname{tr}\left[Q_{0}^{3}\right],
$$

and

$$
\operatorname{tr}\left[I_{3}(Q)\right]=\operatorname{tr}\left[Q_{0} I_{2}(Q)\right]-\frac{1}{3} \operatorname{tr}\left[Q_{0}^{3}\right] .
$$

Since $Q(x)=Q^{*}(x)$, it follows that $\left(\int_{0}^{t} Q\right)^{*}=\int_{0}^{t} Q$. Equation (3.c) yields

or

$$
\operatorname{diag} \int_{0}^{1}\left[\int_{0}^{t} Q(s) d s\right] Q(t) d t=\overline{\operatorname{diag} \int_{0}^{1} Q(t) \int_{0}^{t} Q(s) d s d t}
$$

$$
\operatorname{diag} \int_{0}^{1} \frac{d}{d t}\left[\int_{0}^{t} Q(s) d s\right]^{2} d t=2 \Re\left(\operatorname{diag}\left[I_{2}(Q)\right]\right)=\operatorname{diag}\left[Q_{0}^{2}\right] .
$$

Since $Q_{0}$ is diagonal and real,

$$
\Re\left(\operatorname{tr}\left[Q_{0} I_{2}(Q)\right]\right)=\frac{1}{2} \sum_{k} q_{k}^{3}, \quad \text { and } \quad \Re\left(\operatorname{tr}\left[I_{3}(Q)\right]\right)=\frac{1}{6} \sum_{k} q_{k}^{3} .
$$

In addition

$$
\left|\sum_{k} q_{k}^{3}\right| \leq \sum_{k}\left|q_{k}\right| q_{k}^{2} \leq \sum_{k} \sum_{j}\left[\frac{1}{4}+q_{j}^{2}\right] q_{k}^{2} \leq\left[\frac{K}{4}+\operatorname{tr} I_{2}(Q)\right] \operatorname{tr} I_{2}(Q),
$$

which provides a bound for $\Re\left(\operatorname{tr} I_{3}(Q)\right)$.

When $Q=Q^{*}$,

$$
\operatorname{tr} \int_{0}^{1} Q^{2}=\sum_{i, j} \int_{0}^{1}\left|Q_{i j}\right|^{2}
$$

which is nonnegative. Since $\mathcal{I}_{3}$ is fixed and the real part of $I_{3}(Q)$ is bounded, $\int_{0}^{1} Q^{2}$ is bounded. That is, we have an $L^{2}$ bound for self adjoint matrix potentials $Q(x)$ with a fixed Floquet variety.

The compactness result will follow from bounds on $\int\left[Q^{\prime}\right]^{2}$ based on the invariant $\mathcal{I}_{5}$. Note first that

$$
\int_{0}^{1}\|Q(x)\| \leq C_{1} \int_{0}^{1}\left(\operatorname{tr}\left[Q^{*}(x) Q(x)\right]\right)^{1 / 2} \leq C_{1} \int_{0}^{1}\left(\frac{1}{4}+\operatorname{tr}\left[Q^{2}(x)\right]\right) .
$$

Since $\int_{0}^{1}\|Q(x)\|$ is bounded by some constant $C_{2}$, there is an $x \in[0,1]$ such that $\left\|Q\left(x_{0}\right)\right\| \leq C_{2}$.

A pointwise estimate follows from

$$
\|Q(x)\|=\left\|Q\left(x_{0}\right)+\int_{x_{0}}^{x} Q^{\prime}(t) d t\right\| \leq C(\epsilon)+\epsilon \operatorname{tr} \int_{0}^{1}\left[Q^{\prime}(t)\right]^{2}, \quad \epsilon>0 .
$$

These observations lead to

$$
\sup _{0 \leq x \leq 1}\|Q(x)\| \leq C(\epsilon)+\epsilon \operatorname{tr} \int_{0}^{1}\left[Q^{\prime}\right]^{2}
$$


For self adjoint matrices $Q(x)$ we also have $\|Q(x)\|^{2} \leq C_{3} \operatorname{tr}\left[Q^{2}\right]$. Using the constancy of $\mathcal{I}_{5}$ it is then straightforward to establish bounds on

$$
\operatorname{tr} \int_{0}^{1}\left[Q^{\prime}(x)\right]^{2}
$$

Such estimates imply that the magnitudes of the entries of matrix potentials $Q(x)$ with the same Floquet variety are uniformly bounded, and form an equicontinuous family, which establishes compactness of the closure in the space of continuous matrix valued potentials with the norm $\sup _{0 \leq x \leq 1}\|Q(x)\|$.

\section{REFERENCES}

[1] L. Ahlfors, Complex Analysis, McGraw-Hill, New York, 1966. MR 32:5844

[2] B. Després, The Borg theorem for the vectorial Hill's equation, Inverse Problems 11 (1995), 97-121. MR 96a:34158

[3] A. Finkel, E. Isaacson and E. Trubowitz, An explicit solution of the inverse periodic problem for Hill's equation, SIAM J. Math. Anal. 18 (1987), no. 1, 46-53. MR 88d:34037

[4] C. Fulton and S. Pruess, Eigenvalue and eigenfunction asymptotics for regular SturmLiouville problems, Journal of Mathematical Analysis and Applications, 188 (1994), no. 1, 297-340. MR 96e:34144

[5] J. Garnett and E. Trubowitz, Gaps and bands of one dimensional periodic Schrödinger operators, Comment. Math. Helvetici 59 (1984), 258-321. MR 85i:34004

[6] K. Iwasaki, Inverse problem for Sturm-Liouville and Hill Equations, Annali di Matematica, Pura ed Applicata (1987), 185-206. MR 89d:34053

[7] T. Kappeler, Fibration of the phase space for the Korteweg-De Vries equation, Annales de l'Institute Fourier 41 (1991), no. 1, 539-575. MR 92k:58212

[8] P. Lax, Periodic solutions of the KdV equation, Communications on Pure and Applied Mathematics 28 (1975), 141-188. MR 51:6192

[9] W. Magnus and S. Winkler, Hill's Equation, Dover, New York, 1979. MR 80k:34001

[10] H.P. McKean and P. van Moerbeke, The spectrum of Hill's equation, Inventiones Math. 30 (1975), 217-274. MR 53:936

[11] H.P. McKean and E. Trubowitz, Hill's operator and hyperelliptic function theory in the presence of infinitely many branch points, Communications on Pure and Applied Mathematics 29 (1976), 143-226. MR 55:761

[12] J. Ralston and E. Trubowitz, Isospectral sets for boundary value problems on the unit interval, Ergod. Th. and Dynam. Sys. 8 (1988), 301-358. MR 89m:34035

[13] E. Trubowitz, The inverse problem for periodic potentials, Communications on Pure and Applied Mathematics 30 (1977), 321-337. MR 55:3408

Department of Mathematics, University of Colorado at Colorado Springs, Colorado Springs, Colorado 80933

E-mail address: carlson@castle.uccs.edu 